\title{
Clinical significance of prognostic score based on age, tumor size, and grade in gastric cancer after gastrectomy
}

This article was published in the following Dove Press journal: Cancer Management and Research

\author{
Jingfeng $L u^{1, *}$ \\ Yi Chen ${ }^{1, *}$ \\ Yanxia Liu ${ }^{2, *}$ \\ Junbin Ding' \\ Zhenhao Piao' \\ Weiyan Liu'
}

'Department of General Surgery, Minhang Hospital, Fudan University, Minhang, Shanghai 20II99, People's Republic of China; ${ }^{2}$ Department of Nursing, Minhang Hospital, Fudan University, Minhang, Shanghai 201199, People's Republic of China

*These authors contributed equally to this work
Correspondence: Weiyan Liu; Zhenhao

Piao

Department of General Surgery, Minhang Hospital, Fudan University, 170 Xinsong Road, Minhang, Shanghai 201199, People's Republic of China

$\mathrm{Tel}+862164923400$

Fax +862164923401

Email xiaolu201804@I63.com;

worldmanio@I26.com
Background: Postoperative management and survival of gastric cancer is mainly determined by pathologic TNM stage. However, gastric cancer is a heterogeneity group, and the survival is quite different even when they are in the same TNM stage. Moreover, TNM stage system does not grasp other important clinicopathologic factors to determine the survival. The aim of the present study is to propose and validate prognostic score based on age, tumor size, and grade. Materials and methods: Patients diagnosed with gastric cancer in the Surveillance, Epidemiology, and End Results database from 1988 to 2012 were included in the present study. Kaplan-Meier methods were adopted and multivariable Cox regression models were built for the analysis of long-term survival outcomes and risk factors.

Results: A total of 26,091 eligible patients diagnosed with noncardia gastric cancer were included in the study. In the univariate and multivariate survival analysis, age at diagnosis, tumor grade, and tumor size were validated as independent prognostic factors $(P<0.05)$. Then, we proposed a prognostic score calculated from the number of risk factors, with 0,1 , and 2 points each given for favorable, intermediate, and poor prognostic categories of age $(\leq 50$, $50-70$, and $>70$ ), grade (well, moderate, and poor differentiation), and size $(\leq 3,3-6, \geq 7 \mathrm{~cm})$. The prognostic score was verified as independent predictor in both univariate and multivariate survival analyses $(P<0.001)$. In addition, nomograms on cause-specific survival were established according to prognostic factor and all other significant factors, and c-index was 0.715 (95\% CI: 0.706-0.725).

Conclusion: Prognostic score based on age, tumor size, and grade is an independent predictor of survival after gastrectomy. The novel prognostic score can improve the accuracy of prediction for current TNM stage system. Patients who are with a high prognostic score should undergo extensive follow-up after surgery.

Keywords: gastric cancer, prognostic score, survival analysis

\section{Introduction}

Gastric cancer is one of the deadliest malignancies, ranking as the fourth most common cancer in the world ${ }^{1}$ and the second most common cancer in China. ${ }^{2}$ Surgical resection with extensive lymphadenectomy remains the only curative treatment option for gastric cancer. The postoperative adjunctive therapy and prognosis is mainly decided based on the TNM system, which is comprehensively evaluated based on the depth of tumor invasion and the presence of lymph node metastasis and distant metastasis. ${ }^{3}$ However, gastric cancer is a heterogeneity group, and the survival is quite different even when they are in the same TNM stage. Moreover, TNM stage system does not grasp other important clinicopathologic factors to determine the survival. 
Several studies have demonstrated the clinical significance of some common factors as valuable predictors. Tumor size is significantly correlated with gastric cancer progression, and can be regarded as a reliable prognostic factor after gastrectomy. It can supply TNM stage system to improve the prognostic prediction accuracy for gastric cancer patients. ${ }^{4,5}$ Moreover, age and tumor grade have been validated as independent prognostic markers in patients with resectable gastric cancer. ${ }^{6-8}$ However, few reports have investigated tumor depth, tumor grade, and tumor size simultaneously as predictive markers for prognosis in patients with gastric cancer after surgery.

In the present study, we first use the Surveillance, Epidemiology, and End Results (SEER) population-based cohort to investigate the prognostic value of tumor size, tumor grade, and age in gastric cancer and then develop a prognostic score based on these three factors in reference with previously published study to improve accuracy of prognostic prediction in gastric cancer.

\section{Materials and methods}

\section{Data collection}

The SEER database and SEER-stat software (SEER*Stat 8.3.5) were used to search patients diagnosed with gastric cancer released on March 6, 2018. The inclusion criteria were pathologic diagnosis with gastric adenocarcinoma, mucinous adenocarcinoma, and signet ring cell carcinoma; with no distant metastases; receiving surgical resection; having detailed information about the depth of tumor invasion and lymph node metastases; with known tumor size; with intact follow-up information. The years were limited from 1988 to 2012. Patients diagnosed after 2012 were excluded from the study to guarantee sufficient follow-up time. Figure 1 depicts the flowchart of the selection.

Years of diagnosis, sex, race, marital status, grade, histotype, and surgery performed or not, depth of tumor invasion, lymph node status, survival months, and cause-specific survival (CSS) were extracted from the SEER database. All patients were restaged according to eighth TNM stage.

\section{Ethical approval}

According to the Ethical Committee and Institutional Review Board of the Minhang Hospital, Fudan University on Research involving Human Subjects, this type of study does not require approval from an ethics committee in our institute. All procedures were conducted in accordance with the Helsinki Declaration of 1964 and later versions. ${ }^{9}$ All patients were de-identified.

\section{Statistical analysis}

Age and tumor size were first treated as continuous variables, and incorporated into univariate and multivariable Cox regression analyses. After they were validated as independent prognostic factors, they were classified as classification variables. Age was divided into three subgroups by cutoff 50 and 70 years. X-tile program ${ }^{10}$ was performed to select the most appropriate cutoff points for tumor size to stratify patients at different risk of cancer-related death. Analyses of years

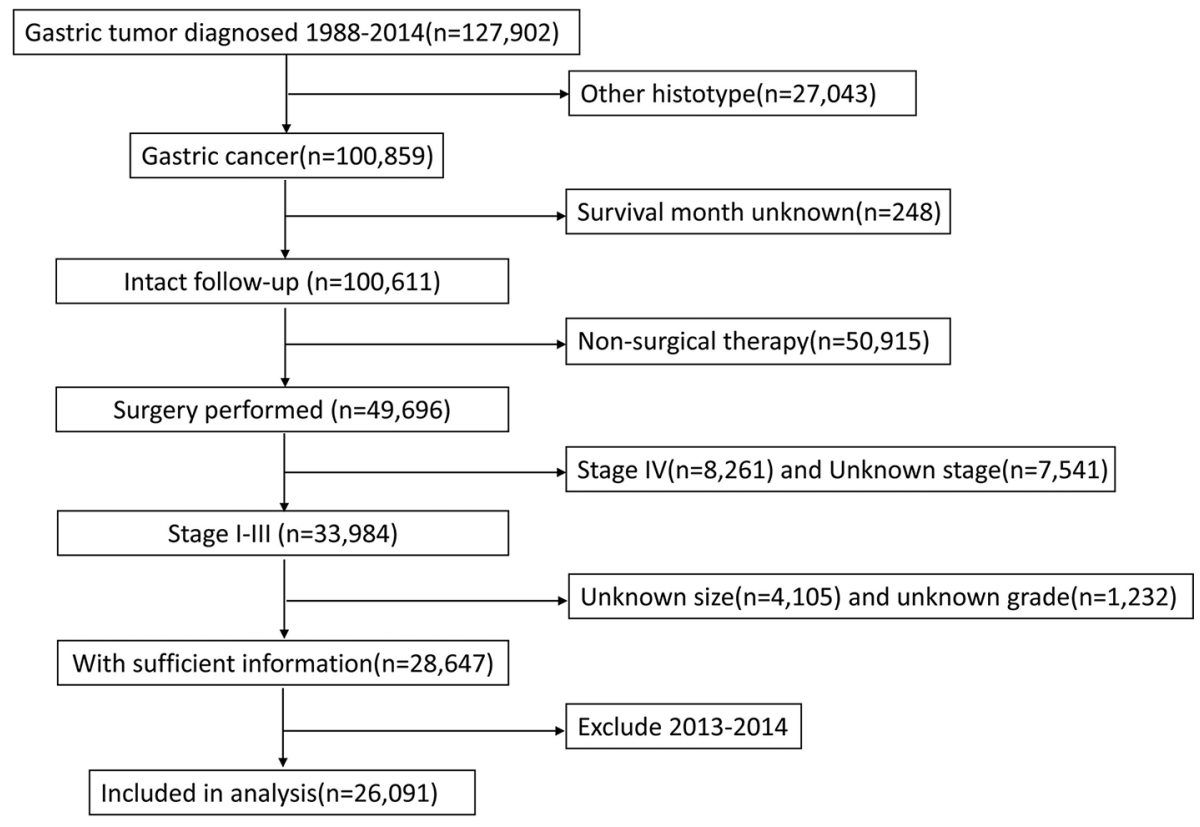

Figure I Flowchart of patients' selection in Surveillance, Epidemiology, and End Results database. 
of diagnosis were performed for five consecutive periods of 5 years, from 1988-1992 to 2008-2012.

The prognostic score was constructed with three patient and tumor characteristics (age at diagnosis, tumor grade, and tumor size) as previously described, ${ }^{11,12}$ and detailed information has been demonstrated in Figure 2. The total score was calculated by summing up the scores of age, grade, and tumor size together.

The primary endpoint of the study was CSS. The 5-year CSS was calculated by the Kaplan-Meier method. The differences in survival between the groups were compared by the log-rank test. Variables that seemed to be significantly associated with survival on univariate analysis were entered into multivariate analysis, which was performed with Cox proportional hazard model. $P$-value $<0.05$ was considered significant. All analyses were conducted using SPSS ${ }^{\circledR}$ version 23 (IBM, Armonk, NY, USA).

Nomograms for possible prognostic factors associated with CSS were established by R software, and the model performance for predicting outcome was evaluated by Harrell's concordance index (c-index) ${ }^{13,14}$ which is a measure of discrimination. The maximum value of the c-index is 1.0 ,

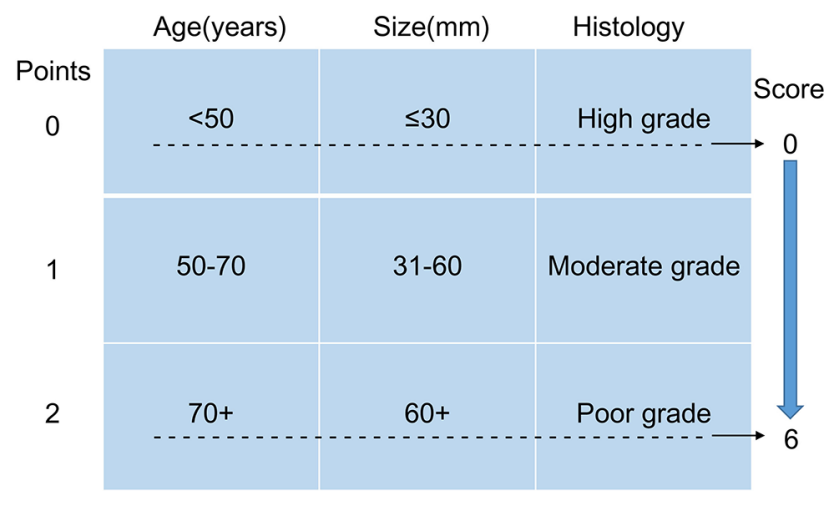

Figure 2 Patient prognostic score: risk stratification. Notes: Data from Smith et al. ${ }^{12}$ indicating a perfect discrimination, whereas 0.5 indicates a random chance to correctly discriminate outcome. In addition to measuring discriminative capacity by c-index, each model was evaluated with calibration curve in which predicted outcomes vs observed outcomes are graphically depicted, which made it possible to conduct further comparison of accuracy in estimating prognosis.

\section{Results \\ Patients' characteristics}

A total of 26,091 eligible patients diagnosed with noncardia gastric cancer were included in the study, of whom 16,663 were male and 9,428 (36.1\%) were female. There were 7,021 (26.9\%) patients diagnosed at stage I, 7,094 (27.2\%) at stage II, and 11,976 (45.9\%) at stage III disease. The percentage of well-differentiated, moderately differentiated, poorly differentiated, and undifferentiated tumor were $5.4 \%, 30.9 \%$, $61.6 \%$, and $2.1 \%$, respectively. The mean age was 68 years. Median duration of follow-up was 51 months.

\section{Construct prognostic score}

The 5-year CSS of the cohort was 53\%. In the univariate analysis, years of diagnosis, sex, age at diagnosis, race, tumor grade, histologic type, primary tumor size, TNM stage, and marital status were significant risk factors for survival (Table 1). Multivariate analysis with Cox regression was performed and all these factors except histologic type were validated as independent prognostic factors for CSS in gastric cancer after gastrectomy (Table 1).

Then, we used X-tile program to divide the patients into high, middle, and low risk of cancer-related death with optimal cutoff of 3 and $6 \mathrm{~cm}$ in terms of tumor diameters. The 5 -year CSS for patients with tumor diameter $\leq 3,4-6$, and $>6 \mathrm{~cm}$ were $69.9 \%, 48.1 \%$, and $39.9 \%$, respectively, and the

Table I Univariate and multivariate survival analyses for evaluating the clinicopathologic characteristics on gastric cancer cause-specific survival in SEER database

\begin{tabular}{|c|c|c|c|c|}
\hline \multirow[b]{2}{*}{ Variable } & \multicolumn{2}{|l|}{ Univariate analysis } & \multicolumn{2}{|c|}{ Multivariate analysis } \\
\hline & HR (95\% Cl) & $P$-value & HR (95\% Cl) & $P$-value \\
\hline Years of diagnosis & $0.976(0.974-0.979)$ & $<0.001$ & $0.992(0.989-0.995)$ & $<0.001$ \\
\hline Sex & $0.897(0.862-0.932)$ & $<0.001$ & $0.870(0.835-0.907)$ & $<0.001$ \\
\hline Age & $1.003(1.002-1.005)$ & $<0.001$ & $1.011(1.009-1.012)$ & $<0.001$ \\
\hline Race & $0.873(0.852-0.893)$ & $<0.001$ & $0.896(0.875-0.917)$ & $<0.001$ \\
\hline Grade & $1.473(1.428-1.520)$ & $<0.001$ & $1.155(1.116-1.196)$ & $<0.001$ \\
\hline Histotype & 1.095 (I.070-I.I20) & $<0.001$ & $1.015(0.991-1.040)$ & 0.226 \\
\hline Size & 1.140 (I.133-I.148) & $<0.001$ & 1.021 (1.014-1.029) & $<0.001$ \\
\hline TNM stage & I.497 (I.48I-I.5I4) & $<0.001$ & I.47I (I.453-I.490) & $<0.001$ \\
\hline Marital status & $1.014(1.005-1.023)$ & 0.003 & $1.025(1.015-1.035)$ & $<0.001$ \\
\hline
\end{tabular}

Abbreviation: SEER, Surveillance, Epidemiology, and End Results. 
difference was statistical $\left(\chi^{2}=1443.42, P<0.001\right)$ (Figure 3 ). We also used cutoff 50 and 70 years to divide patients into three subgroups with score $0-2$ based on prior cohort studies concerning the prognostic factors of age. ${ }^{15,16}$ Patients with poorly and undifferentiated tumor were gathered together and were scored 2 , and those with well and moderately differentiated were scored 0 and 1 , respectively.

After summing up the score from age, tumor grade, and tumor size, we generate total score. There were $0.2 \%$ patients in score 0 subgroup, $2.2 \%$ in score 1 subgroup, $10.4 \%$ in score 2 subgroup, $23.6 \%$ in score 3 subgroup, $31.5 \%$ in score 4 subgroup, $23.0 \%$ in score 5 subgroup, and $9.1 \%$ in score 6 subgroup. For there were only $0.2 \%$ patients with score 0 , we gathered scores 0 and 1 together to generate new score 1 .

\section{Prognostic significance of prognostic score}

There was a gradual decrease in CSS as prognostic score increased. The 5-year CSS for patients with 1, 2, 3, 4, 5, and 6 score were $82.2 \%, 71.4 \%, 62.0 \%, 51.1 \%, 41.6 \%$, and $35.5 \%$, respectively, and the difference was statistically significant $(P<0.001)$ (Table 2 , Figure 4). Then, a multivariate Cox regression model was conducted to identify the variables that were independently associated with CSS in gastric cancer and it was found that years of diagnosis, sex, race, TNM stage, marital status, and prognostic score are independent prognostic factors in patients with gastric cancer, and the HRs gradually increased as the score increased $(P<0.05)$ (Table 2$)$.

\section{Development and validation of nomograms for predicting prognosis for gastric cancer}

To predict CSS of patients with gastric cancer after gastrectomy, nomograms were established by multivariate Cox regression model according to all significantly independent factors for CSS (Figure 5A). Nomograms can be interpreted by summing up the points assigned to each variable, which is indicated at the top of the scale. The total points can be converted to predict 5-year probability of death and recurrence or metastasis for a patient in the lowest scale. ${ }^{14,17}$ The Harrell's c-indexes for CSS were 0.715 (95\% CI: 0.706-0.725), which is higher than the TNM stage alone (c-indexes 0.701, 95\% CI: 0.692-0.715). Calibration curves for the nomograms (Figure 5B) revealed no deviations from the reference line and no need of recalibration.

\section{Discussion}

In our large population-based cohort study, we first assessed the clinical significance of age at diagnosis, tumor size, and grade for predicting prognosis in patients with stage I-III gastric cancer. We then proposed a prognostic score as a new predictor calculated solely from age at diagnosis, tumor size, and grade. Finally, we evaluated the clinical significance of prognostic score as a predictor of survival and developed nomogram-incorporated prognostic score and TNM stage to improve the accuracy of the prediction of survival in patients with stage I-III gastric cancer.

Age plays a significant role in gastric cancers. Age at diagnosis was not only used as an indispensable adjusted element in the observational or interventional studies, but also contained inestimable value for prognosis. A population-based study including 7,762 patients with operable gastric cancer indicated that patients diagnosed at the age of $<45$ years had the lowest HR of CSS and the risk of death increased with age, being the highest for patients older than 76 years ${ }^{16}$. The worse survival for the elderly might be explained partly by inadequate treatment. ${ }^{18} \mathrm{~A}$ bad performance status cannot tolerate the extensive lymphadenectomy and standardized

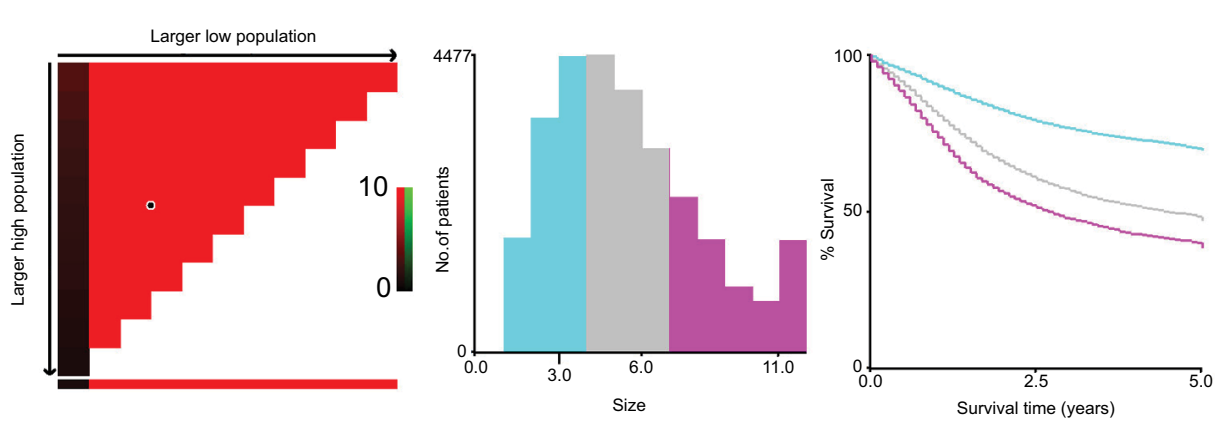

Figure 3 X-tile analyses of 5-year cause-specific survival were performed by using patients' data to determine the optimal cutoff values for tumor size. Notes: Tumor size was treated as category variable for each $1 \mathrm{~cm}$. The sample of gastric cancer patients was equally divided into training and validation sets. X-tile plots of training sets are shown in the left panels, with plots of matched validation sets shown in the smaller inset. The optimal cutoff values highlighted by the black circles in left panels are shown in histograms of the entire cohort (middle panels), and Kaplan-Meier plots are displayed in right panels. $P$-values were determined by using the cutoff values defined in training sets and applying them to validation sets. The optimal cutoff values were 3 and $6 \mathrm{~cm}(P<0.00 \mathrm{I})$. 
Table 2 Multivariate survival analysis for evaluating the prognostic score on gastric cancer cause-specific survival in SEER database

\begin{tabular}{|c|c|c|c|c|}
\hline \multirow[t]{2}{*}{ Variable } & \multirow[t]{2}{*}{$\mathbf{N}$} & \multirow[t]{2}{*}{ 5-year CCS (\%) } & \multicolumn{2}{|l|}{ Multivariate analysis } \\
\hline & & & HR $(95 \% \mathrm{Cl})$ & $P$-value \\
\hline Years of diagnosis & & & & $<0.001$ \\
\hline 1988-1992 & 2,339 & 44.8 & Reference & \\
\hline 1993-1997 & 3,575 & 47.2 & $0.929(0.864-0.998)$ & 0.044 \\
\hline 1998-2002 & 5,709 & 50.0 & $0.928(0.868-0.993)$ & 0.030 \\
\hline 2003-2007 & 7,425 & 55.3 & $0.87 I(0.8 I 5-0.930)$ & $<0.001$ \\
\hline $2008-2012$ & 7,043 & 61.0 & $0.808(0.754-0.867)$ & $<0.001$ \\
\hline Sex & & & & $<0.001$ \\
\hline Male & 16,663 & 51.8 & Reference & \\
\hline Female & 9,428 & 56.1 & $0.874(0.838-0.910)$ & \\
\hline Race & & & & $<0.001$ \\
\hline White & 17,457 & 51.2 & Reference & $<0.001$ \\
\hline Black & 3,203 & 52.1 & $0.979(0.924-1.037)$ & 0.468 \\
\hline Other $^{\mathrm{a}}$ & 5,431 & 60.8 & $0.789(0.735-0.839)$ & $<0.001$ \\
\hline Histotype & & & & 0.713 \\
\hline Adenocarcinoma & 20,274 & 54.7 & Reference & \\
\hline Mucinous & 971 & 47.8 & $0.996(0.907-1.093)$ & 0.925 \\
\hline Signet ring cell & 4,846 & 49.0 & $1.019(0.973-1.069)$ & 0.422 \\
\hline TNM stage & & & & $<0.001$ \\
\hline IA & 4,303 & 87.7 & Reference & \\
\hline IB & 2,718 & 76.5 & $1.69 \mid(1.512-1.890)$ & $<0.001$ \\
\hline IIA & 3,782 & 66.0 & $2.401(2.174-2.652)$ & $<0.001$ \\
\hline IIB & 3,312 & 51.8 & $3.740(3.395-4.120)$ & $<0.001$ \\
\hline IIIA & 5,002 & 41.2 & $4.895(4.468-5.363)$ & $<0.001$ \\
\hline IIIB & 4,749 & 26.6 & 7.165 (6.540-7.848) & $<0.001$ \\
\hline IIIC & 2,225 & 13.5 & $10.607(9.611-11.706)$ & $<0.001$ \\
\hline Marital status & & & & $<0.001$ \\
\hline Married & 16,308 & 54.0 & Reference & \\
\hline Unmarried & 9,783 & 52.5 & I.I42 (I.097-I.I89) & \\
\hline Score & & & & $<0.001$ \\
\hline I & 608 & 82.2 & Reference & \\
\hline 2 & 2,715 & 71.4 & 1.389 (I.I44-I.686) & 0.001 \\
\hline 3 & 6,165 & 62.0 & 1.521 (1.263-I.833) & $<0.001$ \\
\hline 4 & 8,224 & 51.1 & $1.717(1.427-2.066)$ & $<0.001$ \\
\hline 5 & 5,998 & 41.6 & $1.932(1.603-2.327)$ & $<0.001$ \\
\hline 6 & 2,381 & 35.5 & $2.201(1.818-2.665)$ & $<0.001$ \\
\hline
\end{tabular}

Note: ${ }^{a}$ Other includes American Indian/Alaska native, Asian/Pacific Islander, and unknown.

Abbreviation: SEER, Surveillance, Epidemiology, and End Results.

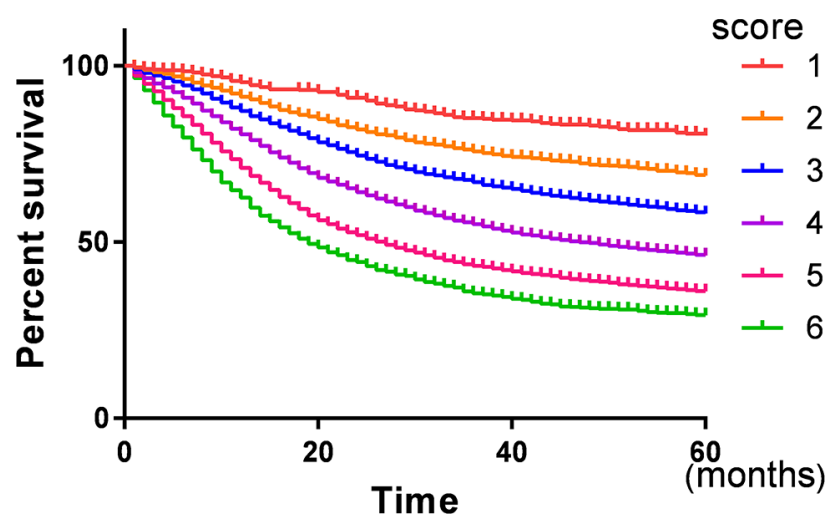

Figure 4 Survival curve for the different prognostic scores.

Notes: Kaplan-Meier analysis was performed to evaluate the correlation of prognostic score and cause-specific survival. Increased prognostic score was correlated with worse survival $\left(\chi^{2}=1509.64, P<0.001\right)$. chemotherapy ${ }^{19}$ while young patients have a good performance status to quicker return of gastrointestinal function after surgery and to tolerate toxicities of standardized chemotherapy and comprehensive treatment. ${ }^{18,20}$

Tumor differentiation is a well-known prognostic factor in patients with gastric cancer. Adachi et al reported that the overall 5-year survival rate for patients with well-differentiated tumor was higher than that for patients with poor-differentiated gastric cancer ( $76 \%$ vs $67 \%$ ), especially for patients with $\geq 10$ cm tumors ( $42 \%$ vs $14 \%)$, and multivariate analysis indicated that histologic type was one of the independent prognostic factors. ${ }^{21}$ The study from Sun et al demonstrated that the clinical response rate in the better differentiated group was significantly 
A

Points

Year

Sex

TNMstage

Maritalstatus

Score

Total Points

1-year Survival

3-year Survival

5-year Survival
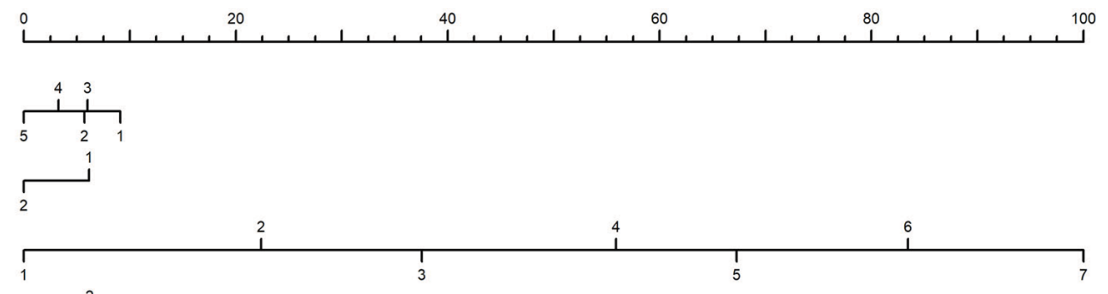

2

1
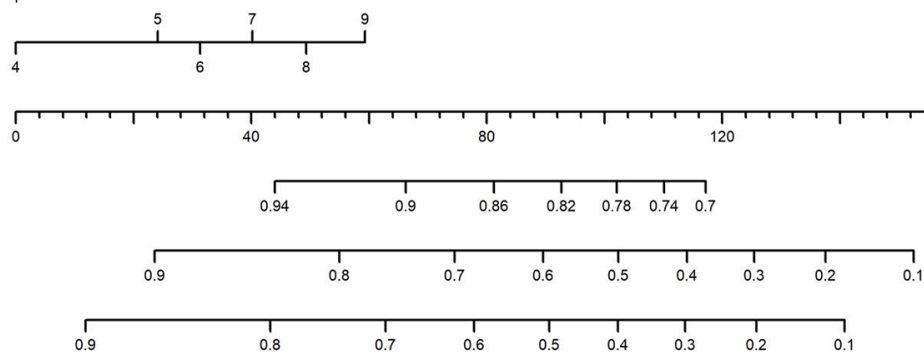

B

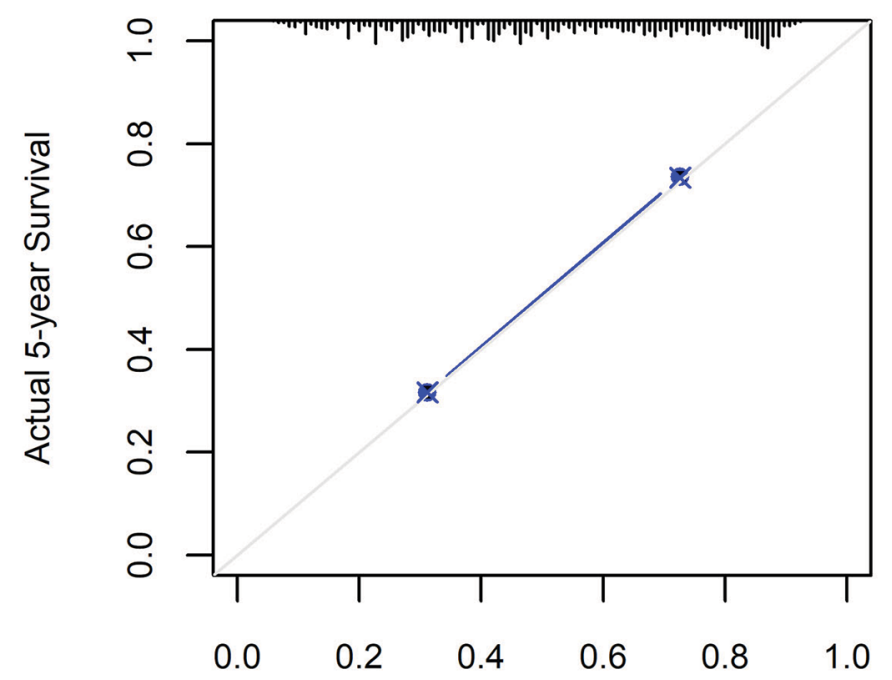

Nomogram Predicted 5-year Survival

Figure 5 Nomograms convey the results of prognostic models using clinicopathologic characteristics and prognostic score to predict cause-specific survival of patients with gastric cancer after gastrectomy.

Notes: $(\mathbf{A}, \mathbf{B})$ Nomograms can be interpreted by summing up the points assigned to each variable, which is indicated at the top of scale. The total points can be converted to predict 5-year probability of death for a patient in the lowest scale. The Harrell's c-index for survival prediction was 0.7 I5 (95\% Cl: $0.706-0.725)$. Calibration curves for 5 -year cause-specific survival using nomograms with clinicopathologic characteristics and prognostic score are shown. The $X$-axis is nomogram-predicted probability of survival and $\mathrm{Y}$-axis is actual survival. The reference line is $45^{\circ}$ and indicates perfect calibration.

higher than that in the poorly differentiated group for patients with locally advanced gastric cancer treated with neoadjuvant chemotherapy. ${ }^{22}$ Not surprisingly, tumor differentiation was significantly associated with poor prognosis in the present study.

We used tumor size as an additional prognostic factor to construct prognostic score in the present study. Many studies have indicated that greater tumor size is closely related with the depth of tumor invasion and the number of lymph node metastasis. ${ }^{5,23,24}$ In the present study, we used X-tile program to divide the cohort into three subgroups, and the risk of cancer-related death increased gradually as tumor size increased, which suggested tumor size as a promising marker for predicting survival.

The most noteworthy point of the current study was that we developed a prognostic score derived from age at diagnosis, tumor size, and grade. Total score was calculated from the number of risk factors, with 0,1 , and 2 points each given for favorable, intermediate, and poor prognostic categories 
of age, grade, and size. Thus, total scores ranged from a minimum of 0 to a maximum of 6 , with the best predicted prognosis associated with the lowest scores and the worst predicted prognosis the highest scores. ${ }^{12}$ The score was verified as an independent prognostic factor in multivariate Cox regression analysis. Importantly, the nomogram-included prognostic score has improved the accuracy of prediction when compared to TNM stage alone.

\section{Limitations}

Although we get the results from a large population-based study, our study still has some limitations. First, this was a retrospective study, which may have some inherent limitation. Second, the information regarding the use of neoadjuvant or adjuvant chemotherapy is missing. It is possible that the tumor may become small if patients received neoadjuvant therapy. And patients who received adjuvant chemotherapy may improve survival than those who rejected chemotherapy, resulting in a potential confounder in this study. Third, the quality of surgery is unknown, especially for those who underwent palliative resection, it may cause apparent confound to the current study. But for the patients' number is very large and the study is more like a real-world study, the above limitations may have limited impact on our results.

\section{Conclusion}

In the current large population-based study, we proposed a prognostic score based on age, tumor size, and grade, and validated the score as an independent predictor of survival after gastrectomy. The novel prognostic score can improve the prediction accuracy for the current TNM stage system. Patients who are with a high prognostic score should undergo extensive follow-up after surgery.

\section{Acknowledgments}

The authors acknowledge the efforts of the Surveillance, Epidemiology, and End Results (SEER) Program tumor registries in the creation of the SEER database. The interpretation and reporting of these data are the sole responsibility of the authors.

\section{Disclosure}

The authors report no conflicts of interest in this work.

\section{References}

1. Torre LA, Bray F, Siegel RL, et al. Global cancer statistics, 2012. $C A$ Cancer J Clin. 20122015;65(2):87-108.

2. Chen W, Zheng R, Baade PD, et al. Cancer statistics in China, 2015. CA Cancer J Clin. 2016;66(2):115-132.
3. Buyyounouski MK, Choyke PL, Kattan M. Prostate Amin MB, editors. AJCC Cancer Staging Manual. 8th ed. Chicago: Springer; 2017:715-726.

4. Zu H, Wang F, Ma Y, Xue Y. Stage-stratified analysis of prognostic significance of tumor size in patients with gastric cancer. PLoS One. 2013;8(1):e54502.

5. Zhao LY, Zhang WH, Chen XZ, et al. Prognostic significance of tumor size in 2405 patients with gastric cancer: a retrospective cohort study. Medicine. 2015;94(50):e2288.

6. Chiaravalli AM, Klersy C, Vanoli A, Ferretti A, Capella C, Solcia E. Histotype-based prognostic classification of gastric cancer. World $J$ Gastroenterol. 2012;18(9):896-904.

7. Schlesinger-Raab A, Werner J, Friess H, Hölzel D, Engel J. Age and outcome in gastrointestinal cancers: a population-based evaluation of oesophageal, gastric and colorectal cancer. Visc Med. 2017;33(4):245-253.

8. Fujiwara Y, Fukuda S, Tsujie M, et al. Effects of age on survival and morbidity in gastric cancer patients undergoing gastrectomy. World $J$ Gastrointest Oncol. 2017;9(6):257-262.

9. Rickham PP. Human experimentation. Code of Ethics of the World Medical Association. Declaration of Helsinki. Br Med J. 1964;2(5402): 177.

10. Camp RL, Dolled-Filhart M, Rimm DL. X-tile: a new bio-informatics tool for biomarker assessment and outcome-based cut-point optimization. Clin Cancer Res. 2004;10(21):7252-7259.

11. Sagara Y, Freedman RA, Vaz-Luis I, et al. Patient prognostic score and associations with survival improvement offered by radiotherapy after breast-conserving surgery for ductal carcinoma in situ: a populationbased longitudinal cohort study. J Clin Oncol. 2016;34(11):1190-1196.

12. Smith GL, Smith BD, Haffty BG. Rationalization and regionalization of treatment for ductal carcinoma in situ of the breast. Int $J$ Radiation Oncol Biol Phys. 2006;65(5):1397-1403.

13. Altman DG, Vergouwe Y, Royston P, Moons KG. Prognosis and prognostic research: validating a prognostic model. BMJ. 2009;338:b605.

14. Li Y, Jia H, Yu W, et al. Nomograms for predicting prognostic value of inflammatory biomarkers in colorectal cancer patients after radical resection. Int J Cancer. 2016;139(1):220-231.

15. Liang YX, Deng JY, Guo HH, et al. Characteristics and prognosis of gastric cancer in patients aged $\geq 70$ years. World J Gastroenterol. 2013;19(39):6568-6578.

16. Chen J, Chen J, Xu Y, et al. Impact of age on the prognosis of operable gastric cancer patients: an analysis based on SEER database. Medicine. 2016;95(24):e3944.

17. Iasonos A, Schrag D, Raj GV, Panageas KS. How to build and interpret a nomogram for cancer prognosis. J Clin Oncol. 2008;26(8): $1364-1370$

18. Nelen SD, Verhoeven RHA, Lemmens V, de Wilt JHW, Bosscha K. Increasing survival gap between young and elderly gastric cancer patients. Gastric Cancer. 2017;20(6):919-928.

19. Lim JH, Lee DH, Shin CM, et al. Clinicopathological features and surgical safety of gastric cancer in elderly patients. J Korean Med Sci. 2014;29(12):1639-1645.

20. Kubota T, Hiki N, Sano T, et al. Prognostic significance of complications after curative surgery for gastric cancer. Ann Surg Oncol. 2014;21(3): 891-898.

21. Adachi Y, Yasuda K, Inomata M, Sato K, Shiraishi N, Kitano S. Pathology and prognosis of gastric carcinoma: well versus poorly differentiated type. Cancer. 2000;89(7):1418-1424.

22. Sun LB, Zhao GJ, Ding DY, et al. Comparison between better and poorly differentiated locally advanced gastric cancer in preoperative chemotherapy: a retrospective, comparative study at a single tertiary care institute. World J Surg Oncol. 2014;12:280.

23. Rio PD, Viani L, Bertocchi E, et al. The prognostic role of tumor size in patients with gastric cancer. Ann Ital Chir. 2017;88:478-484.

24. Deng J, Zhang R, Pan Y, et al. Tumor size as a recommendable variable for accuracy of the prognostic prediction of gastric cancer: a retrospective analysis of 1,521 patients. Ann Surg Oncol. 2015;22(2): $565-572$. 


\section{Publish your work in this journal}

Cancer Management and Research is an international, peer-reviewed open access journal focusing on cancer research and the optimal use of preventative and integrated treatment interventions to achieve improved outcomes, enhanced survival and quality of life for the cancer patient. The manuscript management system is completely online and includes a very quick and fair peer-review system, which is all easy to use. Visit http://www.dovepress.com/testimonials.php to read real quotes from published authors.

Submit your manuscript here: https://www.dovepress.com/cancer-management-and-research-journal 